\title{
Editorial for the special issue on Particle Swarm Optimization
}

\author{
Riccardo Poli • Andries Engelbrecht · Jim Kennedy
}

Received: 1 September 2009 / Accepted: 2 September 2009 / Published online: 11 September 2009

(C) Springer Science + Business Media, LLC 2009

The Particle Swarm Optimizer (PSO), one of the pillars of Swarm Intelligence, is a remarkable algorithm for at least two reasons: (a) it has a very simple formulation which makes it easy to implement, apply, extend and hybridize, and (b) it is a constant source of complex and emergent phenomena, which are at the essence of swarm intelligence. Many people around the world are exploring PSOs and their applications.

This journal special issue solicited novel high-quality scientific contributions on Particle Swarm Optimization in the following areas: empirical and theoretical analyses of the dynamics of PSO particles and populations and their modulation by different kinds of social networks; applications of PSO to high-impact areas and real-world problems; innovative studies and algorithms for setting PSO parameters (including adaptive and parameterless PSOs); advanced bare-bones and distribution-based PSOs; PSOs for stochastic, dynamic, multi-objective and combinatorial optimization problems; novel combinations of PSO algorithms with other AI, Computational Intelligence or OR techniques.

Twenty one articles were submitted covering a large subset of these topics. These underwent a very rigorous review process involving in most cases multiple revisions and reviews of each manuscript over a period of over a year. We would like to thank the editor-in-chief, Marco Dorigo, for his continuous encouragement and support over this period, the many reviewers who generously volunteered their time to help us with this special issue, and the

R. Poli (ه)

School of Computer Science and Electronic Engineering, University of Essex, Colchester, UK

e-mail: rpoli@essex.ac.uk

\author{
A. Engelbrecht \\ Department of Computer Science, University of Pretoria, Pretoria, South Africa \\ e-mail: engel@cs.up.ac.za \\ J. Kennedy \\ US Bureau of Labor Statistics, Washington, DC, USA \\ e-mail: kennedy.jim@gmail.com
}


authors who submitted their best work and carefully revised it to meet the high standards of this journal.

The three excellent articles presented in the following pages represent the result of this complex and taxing work (one further article or two which could not be ready in time for the production of this issue will hopefully appear in future issues of the journal). We briefly introduce them below.

After a period of relatively slow development, the theory of PSO is now making significant and rapid progress, and this trend is likely to continue in the future. The article "The PSO family: deduction, stochastic analysis and comparison" by Fernández Martínez and García Gonzalo represents an excellent contribution to PSO theory. The article introduces a continuous model of PSO which can then be used to derive a whole family of PSOs via the use of different types of finite-difference approximations. The different PSOs are also analyzed mathematically to study their regions of stability in parameter space.

PSO is particularly well-suited to explore continuous spaces with a fixed number of dimensions. One important source of innovation in PSO in recent years is the extension of the paradigm to the exploration of more complex search spaces. This is difficult because certain notions, such as the notion of velocity, are not easily extended to such spaces. The article entitled "Density estimation using a new dimension adaptive particle swarm optimization algorithm" by Yan and Osadciw proposes a new variable-dimensionality PSO which can solve problems where finding the number of components of the solution is part of the problem. They demonstrate the effectiveness of the method on the problem of identifying Weibull mixture models.

Another important area of active research is the hybridization of PSOs with other computational intelligence techniques. This is often used to solve complex real-world problems where one technique is typically used to fix the weaknesses of the other. The article "Application of particle swarm optimization and proximal support vector machines for fault detection" by Samanta and Nataraj represents an excellent example of a case where the hybridization leads to much more effective algorithms. The authors in particular have combined support vector machines with both binary and real-valued PSOs for the purpose of identifying normal and defective bearings in rotating machines from time domain vibration signals.

We hope the readers will find these papers as useful and inspirational as we do. 